\title{
Gaseous thermochemical synthesis and characterization of mixed interstitial phases in the Ti-C-O system
}

\author{
Frederik Bojsen Kværndrup ${ }^{1 *}$, Kristian V. Dahl ${ }^{1}$, Kenny Ståhl ${ }^{2}$, Marcel A.J. Somers ${ }^{1}$, Grethe Winther ${ }^{1}$, Thomas L. Christiansen \\ Technical University of Denmark, DK 2800 Kgs. Lyngby, Denmark \\ ${ }^{1}$ Department of Mechanical Engineering \\ ${ }^{2}$ Department of Chemistry \\ *frebok@mek.dtu.dk
}

\begin{abstract}
$\underline{\text { Abstract }}$
Titanium grade 2 thin foils were carbo-oxidized at $800^{\circ} \mathrm{C}$ in a thermogravimetric analyzer in $\mathrm{CO}$ gas for 20 - 100 hours. The synthesized thin foils were characterized by infrared carbon determination for composition analysis, transmission X-ray diffraction (XRD), light optical microscopy (LOM) and nanoindentation. Thermodynamic equilibrium predictions were performed using ThermoCalc. Oxygen and carbon expanded h.c.p. $\alpha$ and $\mathrm{NaCl}$ type $\delta$ carbo-oxide were identified from the XRD patterns. Rietveld refinements of the XRD patterns revealed an increase in expansion of the h.c.p. $\alpha$ lattice from interstitially dissolved C and O: the c/a ratio increases from 1.588 for interstitially free h.c.p. $\alpha$ to 1.607 after 100 hours of exposure. The overall carbon and oxygen content after 100 hours was $4.2 \mathrm{wt} \%$ and $5.5 \mathrm{wt} \%$, respectively; these overall compositions correspond to the two-phase $\alpha+\delta$ region in the calculated ternary phase diagram. The microstructure from LOM revealed large grains of expanded $\alpha$-phase and a surface zone consisting of porous $\delta$ carbo-oxide. The hardness, as determined by indentation, of the expanded $\alpha$ increased linearly with the c/a ratio, resulting in an increase to 11748 MPA after 100 hours exposure from 2193 MPa for the untreated Ti grade 2 .
\end{abstract}

Keywords: Titanium; Transmission X-Ray diffraction; Rietveld refinement; Gaseous carbo-oxidation; Light optical microscopy; Nanoindentation; ThermoCalc

\section{Introduction}

Titanium (Ti) and its alloys are used in applications where high specific strength, corrosion resistance and light weight are required. However, poor tribological properties makes $\mathrm{T} i$ alloys inapt for applications involving wear loads. This can be alleviated by surface hardening of Ti by incorporation of interstitial elements, $\mathrm{C}$, $\mathrm{N}$ and/or $\mathrm{O}$, in the h.c.p. $\alpha$ crystal structure and via formation of interstitial compounds ([1],[2],[3]). The solubility of carbon in $\alpha$ is maximally 0.08 wt $\%$ at $800{ }^{\circ} \mathrm{C}$ [4], higher contents entail formation of $\mathrm{NaCl}$ type $\delta$ carbide. The solubility of oxygen in $\alpha$ is $14.3 \mathrm{wt} \%$ at $800{ }^{\circ} \mathrm{C}$ and is roughly constant from $600-1750{ }^{\circ} \mathrm{C}$, with ordering taking place $\left(\mathrm{Ti}_{2} \mathrm{O}, \mathrm{Ti}_{3} \mathrm{O}\right)$ at a lower temperature [5]. The $\mathrm{NaCl}$ type $\delta$ oxide, $\mathrm{TiO}_{1-\mathrm{X}}$, has a wide homogeneity range and - ostensibly - shows full inter-solubility with the isomorphous compounds $\mathrm{TiC}_{1-\mathrm{X}}$ and $\mathrm{TiN}_{1-\mathrm{X}}[4]$.

The use of thin foils enables fast trough-diffusion of interstitial elements and hence possibility for synthesis of homogenous materials, provided that equilibrium between the solid state and the gas atmosphere is attained ([6],[7],[8],[9]). Hitherto, no systematic work on the Ti-C-O system using thin foils and controlled gas systems has been performed. The present study examines the Ti-C-O system at $800^{\circ} \mathrm{C}$, using a controlled gaseous atmosphere and thin foils. The synthesized materials provide fundamental information on the lattice parameters, phase composition, microstructure and mechanical properties as a function of different loads of $\mathrm{C}$ and $\mathrm{O}$. The obtained fundamental data is a prerequisite for tailored and optimized surface engineering of titanium alloys.

\section{Materials and Methods}

1. Thermochemical synthesis

Annealed $99.6 \%$ pure grade 2 titanium foils (GoodFellow) with a thickness of $25 \mu \mathrm{m}$ were cut into pieces of roughly $1.3 \mathrm{~cm}$ in length and $0.6 \mathrm{~cm}$ in width. A total mass of $640 \mathrm{mg}$ of foil was carbo-oxidized in a Netzsch STA449 C Jupiter thermal analyzer at $800{ }^{\circ} \mathrm{C}$ using a gas flow of $100 \mathrm{ml} / \mathrm{min} \mathrm{CO}(99.97 \%$ purity) and $3 \mathrm{ml} / \mathrm{min} \mathrm{Ar}(99.999 \%$ purity) as protective gas. Heating to $800{ }^{\circ} \mathrm{C}$ was performed with a heating rate of $20^{\circ} \mathrm{C} / \mathrm{min}$; cooling was performed with an average cooling rate of approximately $50^{\circ} \mathrm{C} / \mathrm{min}$, which is the maximum cooling rate of the thermal analyzer. After carbo-oxidizing for 20 hours, the treatment was terminated by cooling to room temperature in the active gas and flushing with Ar before opening. Approximately $50 \mathrm{mg}$ of the treated foils was sampled for further analysis. Subsequently, the remaining foils were carbo-oxidized for another 20 hours (cf. above); this procedure was repeated until a total 100 hours of treatment was reached. The total uptake of carbon and oxygen in the foils was (continuously) recorded by the thermal analyzer.

\section{Carbon content determination}

Determination of carbon content of the 100 hours sample was performed on a CS230 LECO Carbon/Sulfur Determinator using LECO iron chip accelerant and Lecocel II. Quantification was done by conversion of $\mathrm{C}$ to $\mathrm{CO}_{2}$ by $\mathrm{O}_{2}$ and infrared spectrometry. A steel containing $0.832 \mathrm{wt} \% \mathrm{C}$ was used for calibration of the instrument (certified calibration material). No other samples were analyzed due to limited sample quantities.

\section{ThermoCalc predictions}

Thermodynamic equilibrium predictions were performed in Thermo-Calc (TC) version 2017b using the Thermo-Calc Software TCTI1 'Titanium and Titanium Aluminide-based alloys' database version $1[10]$. The database only contains the Ti-C and Ti-O binary interaction terms, and not the full Ti-C-O ternary interaction terms.

\section{X-Ray diffraction and Rietveld refinement}

X-ray diffraction (XRD) was performed with a Huber G670 using a CuK( $\alpha$ )1 source in transmission (Guinier) mode, where the samples were fixed at an angle of $45^{\circ}$ with respect to the incident beam and rotated to improve grain statistics and reduced influence of texture effects. The image strip detector was read out in a $2 \theta$ range of $3-100^{\circ}$ with a step size of $0.005^{\circ}$ after an exposure time of 1 hour. Rietveld refinements of intensity versus scattering angle (20) was performed in WINPOW a modified version of the LHMP program [11], in order to calculate the lattice parameters, phase composition and zero shift corrections. Systematic shifts in the XRD patterns due to slight bending of the foils were corrected for by using the calculated zero shift corrections from WINPOW, where a typical shift was -0.0620 . The background was corrected with Chebyshev polynomials and the peaks were fitted with a pseudoVoigt function. A large degree of preferred orientation (texture) in the c direction (001) of the h.c.p. $\alpha$ structure, necessitated preferred orientation corrections. The texture was also present in the untreated foils, which indicates that the texture is a remnant of the manufacturing of the foils (hot rolling). Residual values of $R_{p} \leq 2.68 \%$ and goodness-of-fit values $\chi^{2} \leq$ 4.56 were obtained, with difference curves of low intensity, indicating insignificant differences between the calculated and measured patterns. Lattice parameter values for the $\alpha$ and $\delta$ phases had minor standard deviation values of below $10^{-5} \AA$.

\section{Microstructure analysis}

Pieces of the treated foils were embedded in epoxy, ground and polished. Etching was performed using Murakami's reagent ( $10 \mathrm{~g}$ potassium ferricyanide, $10 \mathrm{~g}$ sodium hydroxide and 100 $\mathrm{mL}$ water) at $55^{\circ} \mathrm{C}$ for $3.5 \mathrm{~min}$. Light optical microscopy (LOM) was performed on the cross sections using polarized light on a ZEISS Axio Vert.A1 at $50 \mathrm{x}$ magnification.

\section{Mechanical properties from nanoindentation}

(C) The Authors, published by EDP Sciences. This is an open access article distributed under the terms of the Creative Commons Attribution License 4.0 (http://creativecommons.org/licenses/by/4.0/). 
Nanoindentation to evaluate the hardness was performed on the cross-sections of the foils, using a $\mathrm{NHT}^{2}$ from CSM Instruments with a diamond Berkovich indenter, a load of $40 \mathrm{mN}$ and a resulting depth of $450-500 \mathrm{~nm}$ via the Oliver-Pharr indentation method. Approximately 200 indents were made on each sample using an advanced visually assisted matrix mode. Each indent was evaluated for shape, position, unwanted crack formation and their load-displacement curve. The hardness was consequently based on $43-63$ indents on average, due to the narrowness of the foils that made position calibration imprecise and the brittleness in areas of the foils.

\section{Results and Interpretation}

1. Thermogravimetric and combustion analysis

The $\mathrm{CO}$ gas system is characterized by having a low partial pressure of $\mathrm{O}_{2}$ and a high activity of carbon, however the precise values are undefined: The $\mathrm{CO}+\mathrm{CO} \mathrm{O}_{2}$ buffer system (Boudouard reaction) results in a controlled partial pressure of oxygen and carbon activity as a function of temperature provided that the ratio of $\mathrm{CO}$ and $\mathrm{CO}_{2}$ is known. The cumulative carbon and oxygen uptakes of the samples from the thermogravimetric analysis (TGA) are shown in Figure 1. Initially there is a fast uptake of $\mathrm{C}$ and $\mathrm{O}$ with an increase in the wt $\%{ }_{\mathrm{C}}+\mathrm{O}$ to $4.65 \%$ in the first 20 hours, which drops notably in the subsequent hours suggesting that the uptake could be diffusion controlled. This can be evaluated from a (wt $\left.\%{ }^{\mathrm{C}+\mathrm{O}}\right)^{2}$ vs. time (hours) plot in Figure 1 and confirms a parabolic rate law for up to 60 hours and a deceleration after $60 \mathrm{~h}$. The total sample composition after 100 hours was 4.2 (+/- 0.34 ) wt $\% \mathrm{C}$ and 5.5 $(+/-0.33) \mathrm{wt} \% \mathrm{O}$ calculated from the TGA and carbon determination These values represent the combined content in $\delta$ and $\alpha$ in the foils. From the uptake curve, it is clear that saturation of the foils has not been obtained after 100 hours exposure. This implies that no equilibrium has been achieved between the gaseous atmosphere and the solid state.
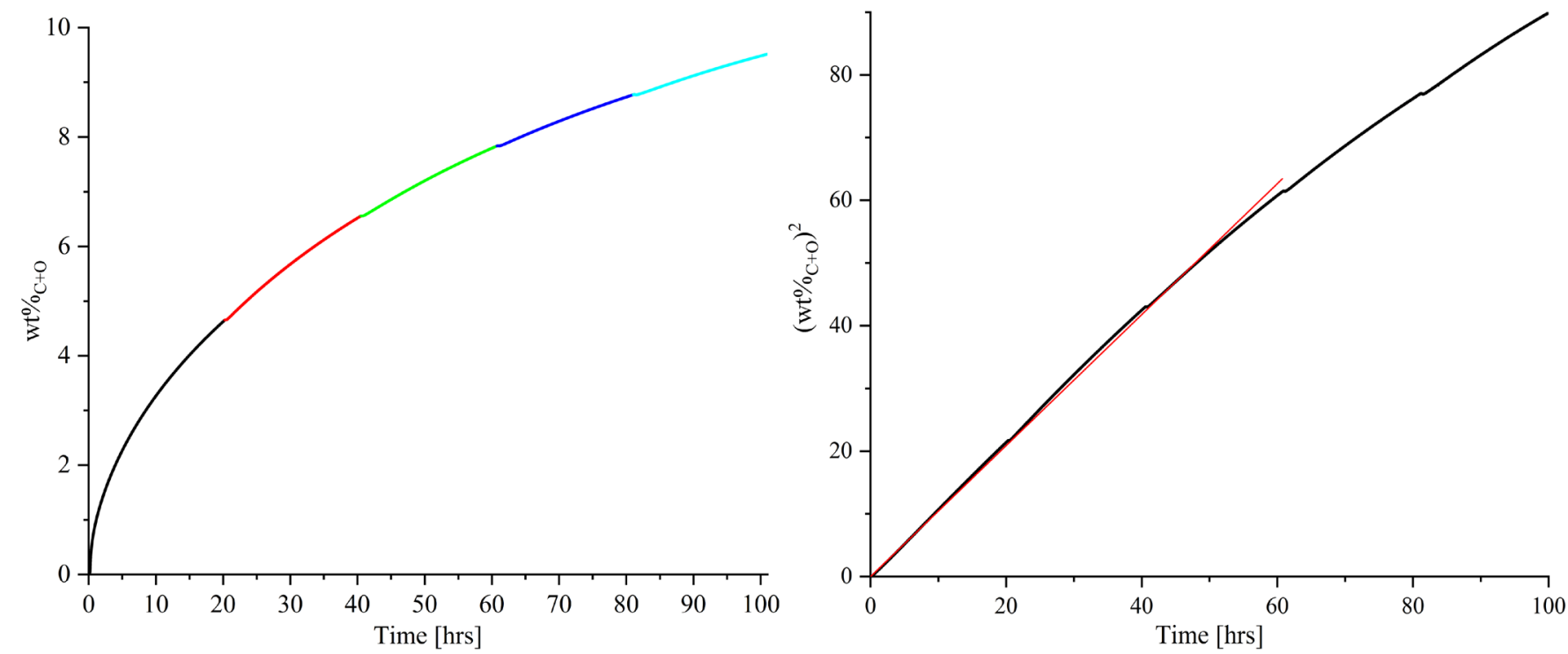

Figure 1: Cumulative thermogravimetric curve for the five samples treated from $20-100$ hours at $800{ }^{\circ} \mathrm{C}$ in $\mathrm{CO}$, corrected for sample removal (left). Plot of (wt $\left.\%{ }^{\mathrm{C}+\mathrm{O}}\right)^{2} \mathrm{vs}$. time (hours) with a linear fit from $0-60$ hours (right).

\section{Light optical microscopy}

The microstructure evolution is presented in Figure 2. The microstructure consists of an outer $\delta$ layer and an inner (C+O expanded) $\alpha$ matrix (cf. XRD in the following section). Evidently, the growth of $\delta$ phase is promoted by grain boundaries; from 40 hours onwards fast growth in these regions is observed. Significant grain growth occurs in the $\alpha$ phase. The difference in color between grains is due to the polarized light, which makes identification of grains with different orientations easier. The notably darker interphase layer between the outer $\delta$ and inner $\alpha$ indicates the presence of another $\delta$ phase (cf. XRD), which has different etching response towards the applied Murakami reagent; this could potentially be attributed to a lower content of carbon.
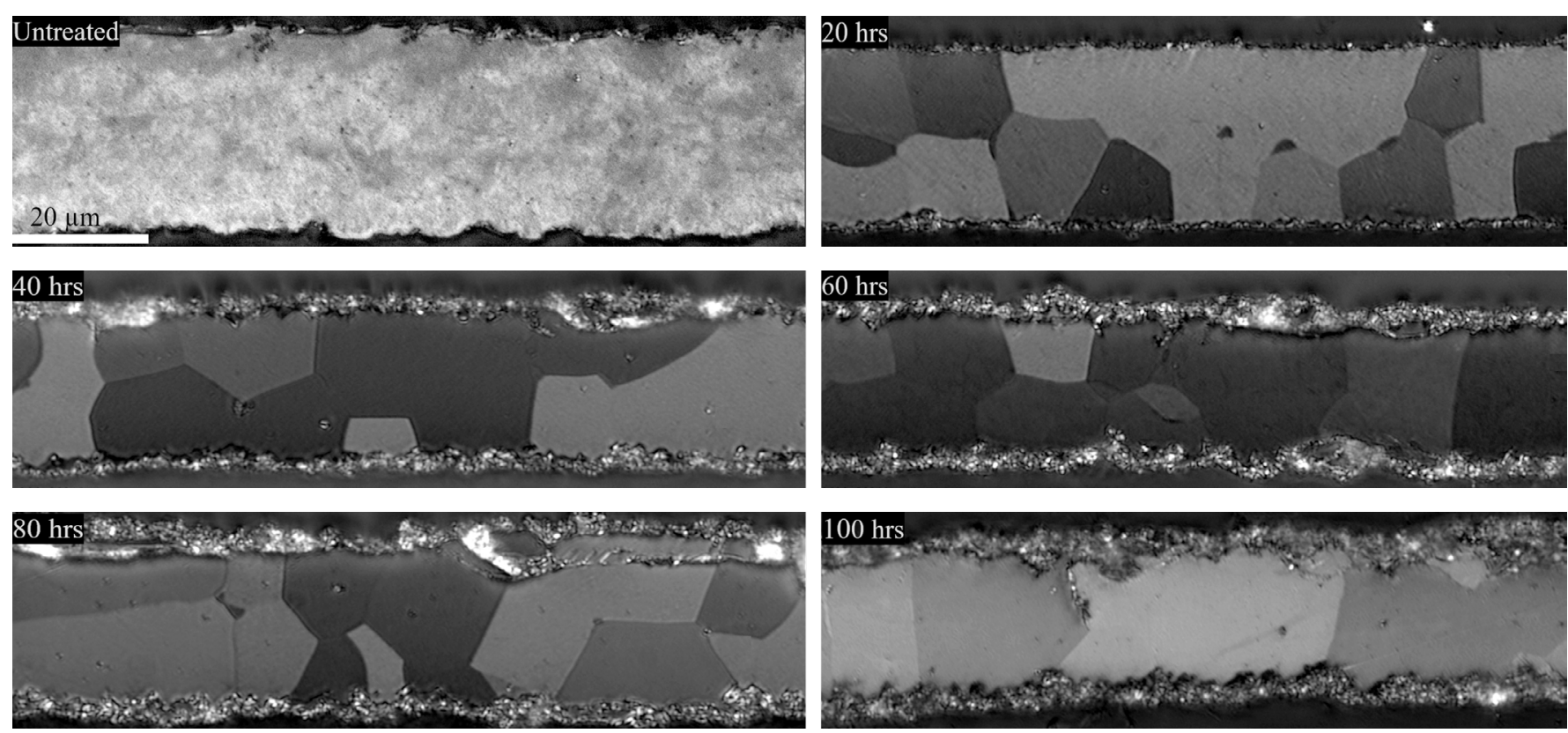

Figure 2: Light optical micrographs obtained by polarized light of Murakami etched cross-sections of the synthesized thin foils. The scale bar given for the untreated reference applies for all micrographs. The small pits in the $\alpha$ phase are most likely residues from spallation of the $\delta$ layer during grinding and polishing. 


\section{Transmission X-ray diffraction}

The transmission XRD pattern of the C-O synthesized foils and the untreated reference foil are given in Figure 3 . The untreated reference consists of $\alpha$ and has a weak [001] texture, while the carbo-oxidized samples are converted into expanded $\alpha$ and $\delta$. The intensity of the $\delta$ peaks increased with treatment time, along with reduction of the intensity of the $\alpha$ peaks, indicating transformation of $\alpha$ into $\delta$ phase. The $\alpha$ peaks that are only sensitive to a change of the $\mathrm{c}$ lattice parameter in the h.c.p. lattice, such as the [002] peak at $38^{\circ} 2 \theta$ or the [012] peak at $52^{\circ} 2 \theta$, display a clear shift to lower $2 \theta$ values with increasing reaction time, indicating a lattice expansion in the c direction in the h.c.p. crystal and thus an increase in the c/a ratio. The a parameter is not affected by the incorporation of oxygen and carbon to the same degree as the c parameter; it only changes from $2.953 \AA$ for interstitially free h.c.p. $\alpha$ to $2.969 \AA$ after 100 hours of exposure, while the c changes from $4.69 \AA$ to $4.77 \AA$. The $\delta$ [111] and [022] peaks contain small satellite peaks to the higher $2 \theta$ side indicated by a black dot, which are identified as belonging to the oxygen rich $\delta_{2}$ phase in the interphase between the outer $\delta$ layer and the inner $\alpha$ grains (cf. LOM).

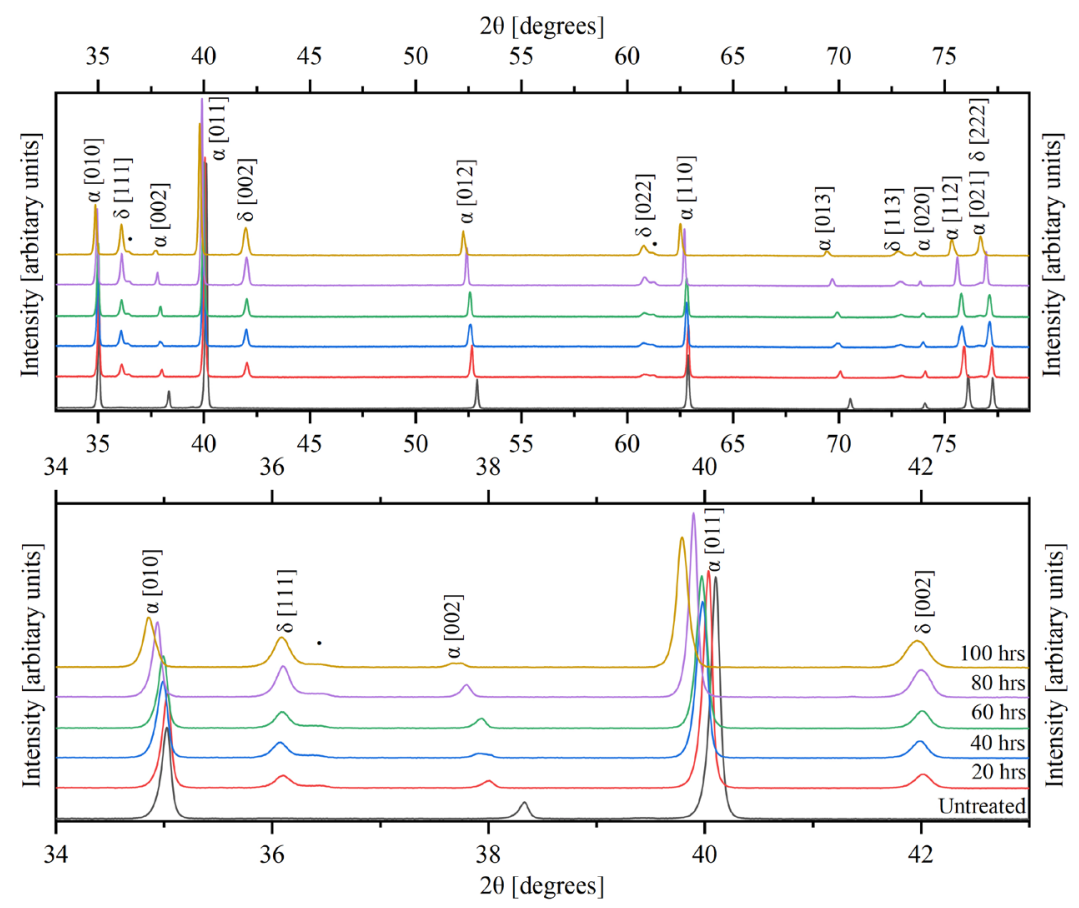

Figure 3: Transmission XRD Pattern showing all peaks from 33 - 7920 (upper) and 34 - 4320 (lower) for untreated and carbo-oxidized samples. Lower pattern sample identification applies to the upper pattern.

\section{Rietveld refinement}

The refined Rietveld profile of the major peaks of the $\alpha$ and $\delta$ phases is shown in Figure 4 for the 100 hours sample. From the residuals curve it is clear that the fit is successful; similar results were obtained for the other samples. The only significant disagreement is at the $\alpha$ [012] peak, which can be explained by the high degree of preferred orientation in the $\mathrm{c}$ direction that can be difficult to fit perfectly. The texture is most likely a consequence of the annealing and rolling of the foils, as preferred orientation is also evident for the untreated reference foil. Minor asymmetry corrections for the $\alpha$ phase for the $20-60$ hours samples were used, due to slight concentration gradients.

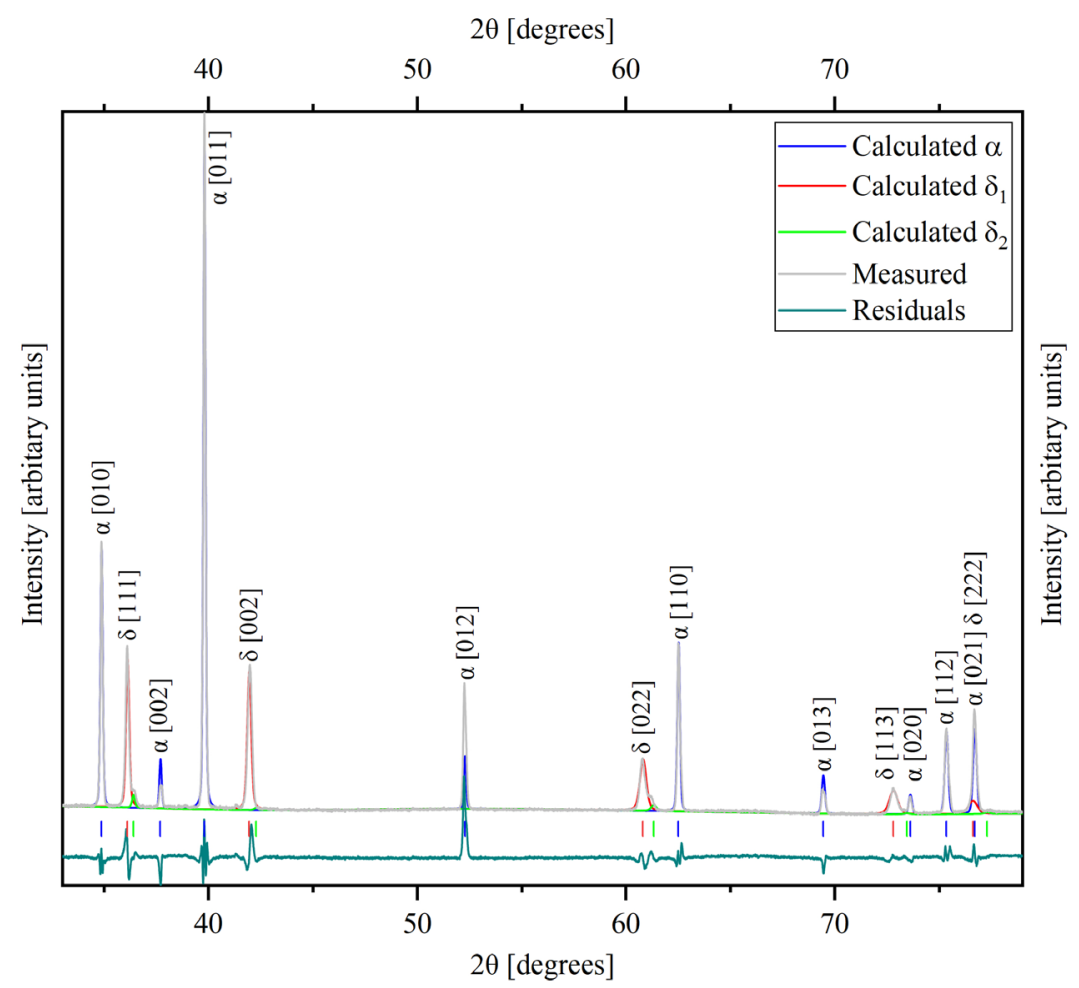


Figure 4: X-ray diffraction pattern, refined Rietveld profile, background curve and difference curve between 33 - 7920 (full range is 3 - $1002 \theta$ ) for the 100 hours sample.

The results from the Rietveld refinement are collected in Figure 5 and show that the total content of interstitials increases with time; the c/a ratio in $\alpha$ increases from 1.6010 to 1.6064 from 20 to 100 hours reaction time, while the untreated foil displayed a c/a ratio of 1.588 . This indicates that the total content of interstitials $(\mathrm{C}+\mathrm{O})$ increases with time, although the specific ratio of the two elements is unknown. Also, a change in ratio between the interstitial elements can lead to a significant change of the c/a ratio as carbon expands $\alpha$ more than oxygen [12]; though this is the behavior of low contents of C and O. The lattice parameters for $\delta_{1}$ and $\delta_{2}$ barely changed with time with averaged values of 4.302 ( $\left.+/-0.002\right) \AA$ and 4.27 $(+/-0.002) \AA$ for $\delta_{1}$ and $\delta_{2}$, respectively. The figures also display the decreasing phase fraction of $\alpha$ and the increasing phase fractions of $\delta_{1}$ and $\delta_{2}$ with reaction time. The wide standard deviations are due to the difficulty of fitting the XRD patterns perfectly due to the texture of $\alpha$, resulting in a severe underestimation of the fraction of $\alpha$ relative to $\delta_{1}$ and $\delta_{2}$, making the results qualitative.

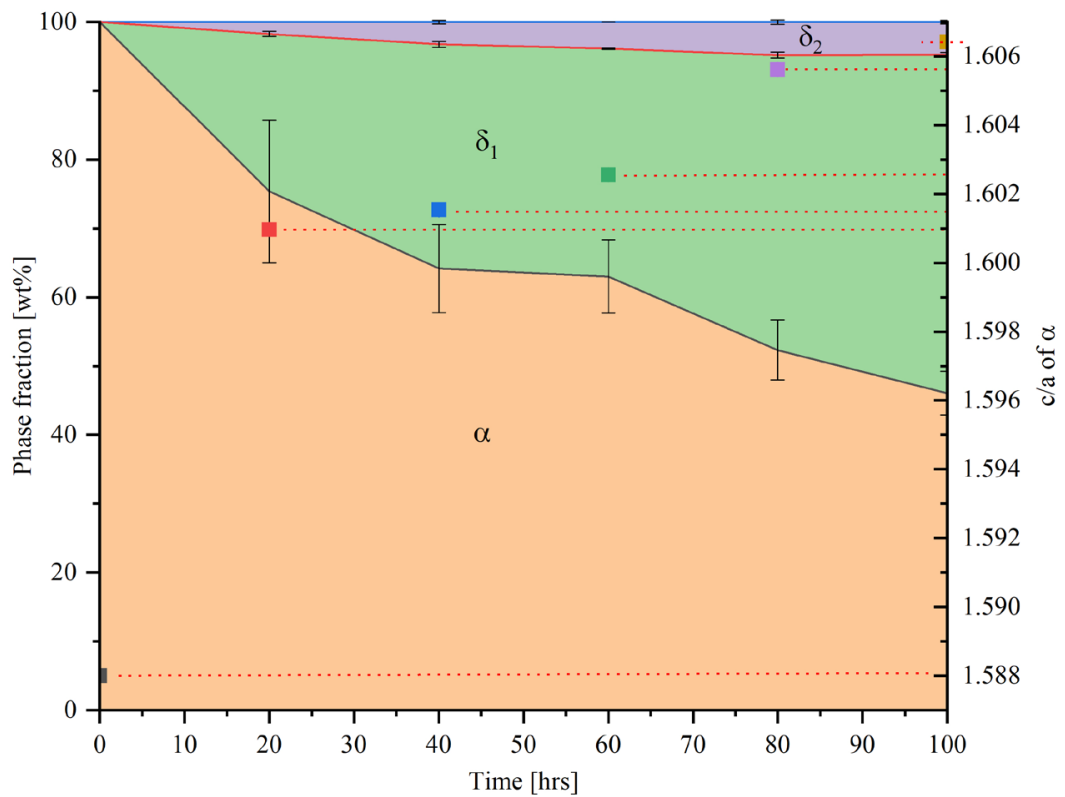

Figure 5: Rietveld refinement phase transformation map showing the phase fraction (wt $\%)$ and c/a ratio of the h.c.p. $\alpha$ lattice vs. reaction time. The fraction of $\delta_{1}$ and $\delta_{2}$ increases along with a decrease of $\alpha$ as a function of reaction time. The concentration of interstitials in $\alpha$ increase with reaction time, leading to an increased c/a ratio. Estimated standard deviations for c/a ratios are too small to be visible.

\section{Nanoindentation}

The hardness, as obtained by nanoindentation, of the expanded $\alpha$ phase is given in Figure 6 . Here the overall tendency is that the hardness increases with reaction time and roughly follows a linear trend with the c/a ratio. This shows that higher concentrations of $\mathrm{C}$ and $\mathrm{O}$ increase the hardness of $\alpha$, however titanium is strongly anisotropic and grain orientation information from electron backscatter diffraction combined with nanoindentation are needed to get the full picture (future work).

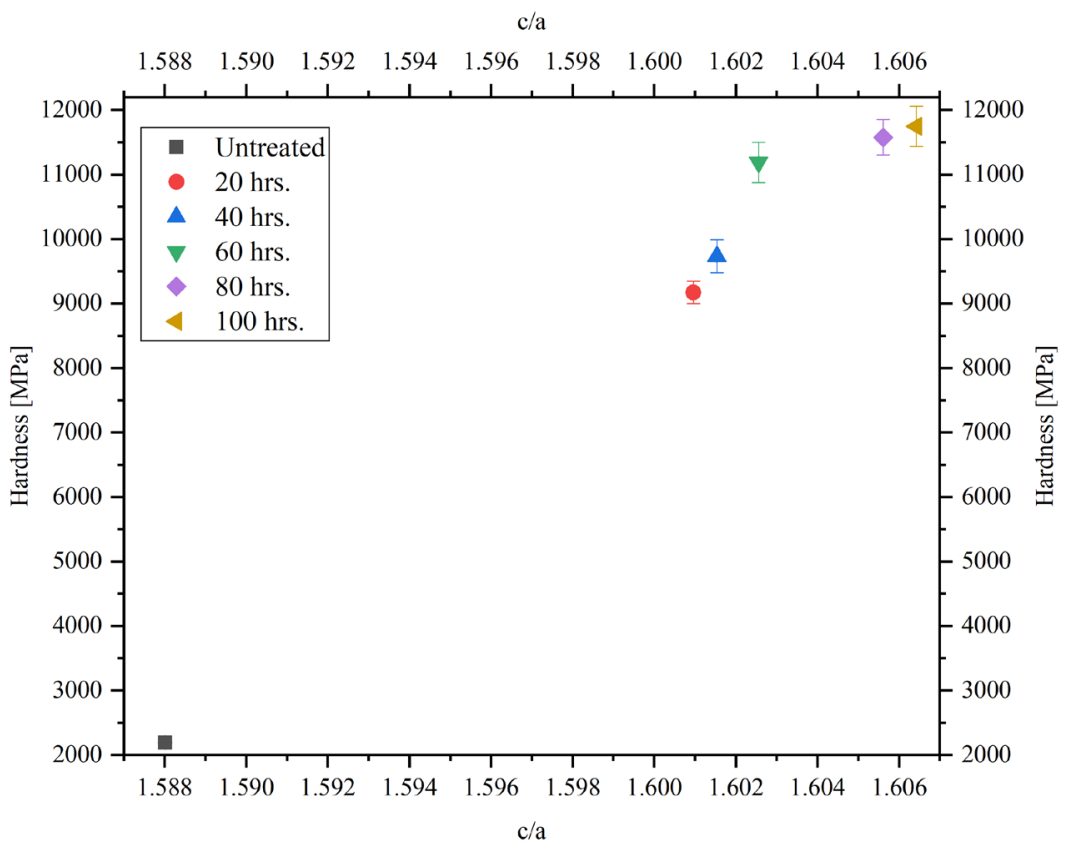

Figure 6: Hardness vs. c/a ratio of $\alpha$ for untreated and carbo-oxidized samples. Based on 43 - 63 validated indents made with a $40 \mathrm{mN} l o a d$ and $400-500 \mathrm{~nm}$ depth.

4. Discussion

1. Thermodynamic equilibrium predictions

Figure 7 displays the ternary Ti-C-O phase diagram at $800{ }^{\circ} \mathrm{C}$ calculated with ThermoCalc. The experimentally determined composition for the 100 hours sample is shown in the diagram, which is within the $\alpha+\delta$ two-phase region. From the cross-sections (Fig. 2) it is obvious that changes in the composition of the $\alpha$ phase rely on diffusion of $\mathrm{O}$ and $\mathrm{C}$ through 
the $\delta$ phase. Consequently, the system has not established an actual equilibrium between the $\alpha$ and $\delta$ phases, as assumed in ThermoCalc. ThermoCalc predicts the existence of a carbon rich $\delta_{1}$ and an oxygen rich $\delta_{2}$ for high concentrations of oxygen, which implies that the thermodynamic data available in the applied database does not consider full inter-solubility between the two compounds. The presence of $\delta_{2}$ phase with a higher concentration of oxygen is consistent with LOM and XRD.

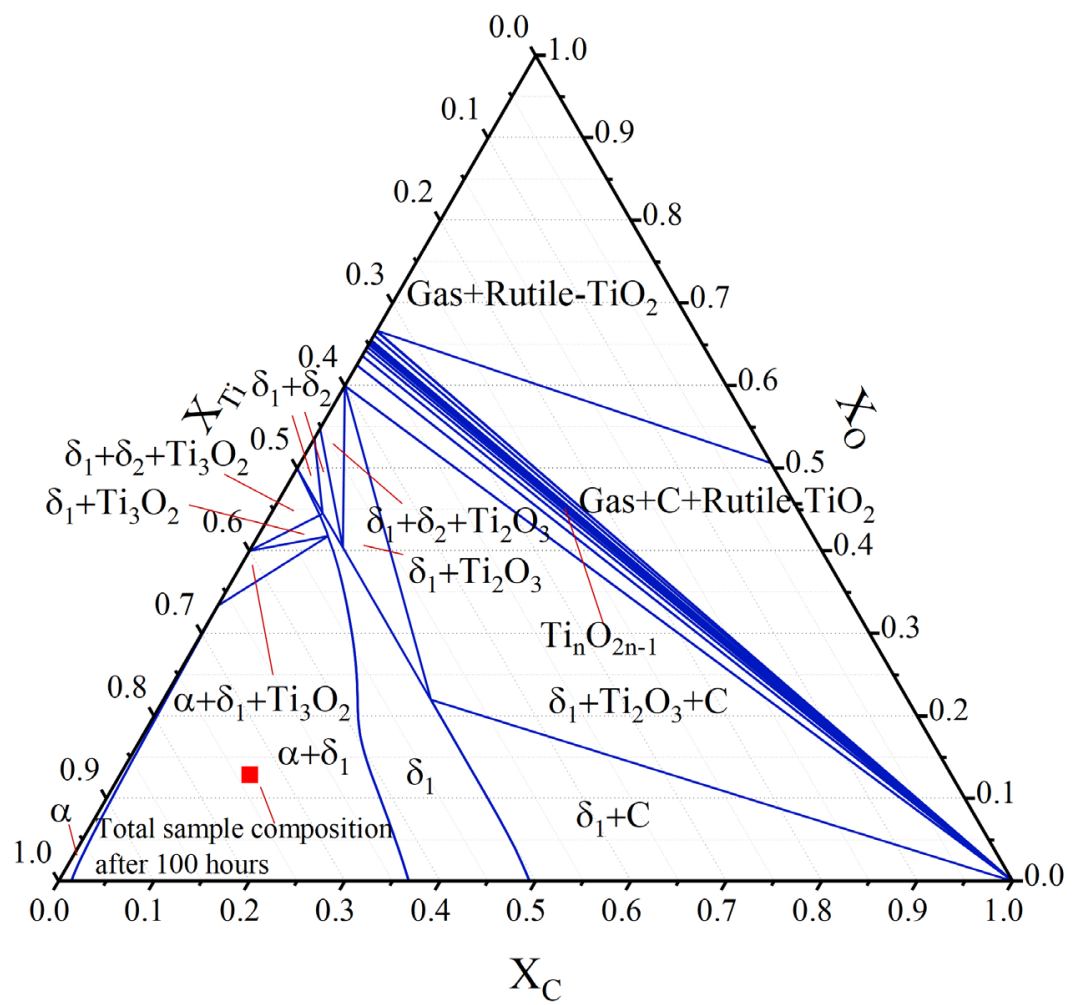

Figure 7: Ternary phase diagram of Ti-C-O at $800{ }^{\circ} \mathrm{C}$. The total sample composition after $100 \mathrm{hrs}$. is based on the TGA total uptake and the LECO carbon determination.

2. Interstitial solubility in $\alpha$; c/a ratio

A potential hypothesis that may explain the increase in the c/a ratio from $60-100$ hours, in the regime where the uptake is slow, relates to the surface reaction and the applied gas species. The atmosphere of flowing $\mathrm{CO}$ results in a very high carbon activity and low partial pressure of oxygen, albeit in principle undefined. The dissociation of CO results in formation of carbon and formation of $\mathrm{CO}_{2}$ at the surface; the surface kinetics of this carburizing reaction is initially fast and adsorbed $\mathrm{C}$ can only enter into solid solution (or remain at the surface and form graphite). Carbon in the solid state is not able to leave the material by reaction with CO. Conversely, incorporation of oxygen from a low and undefined partial pressure of oxygen is slower, but oxygen can desorb or be retracted from the solid state by reaction with flowing CO. The initial introduction of carbon, which has a low solubility in $\alpha$, leads to fast formation of a $\delta$ compound layer. The carbon stabilized $\delta$ phase, inhibits a fast exchange of $\mathrm{C}$ and $\mathrm{O}$ in $\alpha$ phase with the gas due to slow diffusion in this phase. Hence, no equilibrium is attained between $\alpha$ and the gas, due to a slow diffusion flux through the $\delta$. The composition of $\alpha$ changes with time due to dissolution of more $\mathrm{O}$, which leads to an increase in c/a ratio and hardness. The sharp increase in c/a ratio from $60-100$ hours coincides with the sharp increase in the fraction of $\delta$ phase. This implies that carbon is expelled from $\alpha$ and results in formation of more carbon-rich $\delta$. The redistribution of interstitials between the two phases is expectedly a slow process. The small variation in the lattice parameter for $\delta$ suggests that $\mathrm{C}$ and $\mathrm{O}$ have very similar expansion effects on the denser $\delta$. For $\alpha$ there is most likely a difference in the degree of expansion, though the extent is unknown at high concentrations $[12$.

\section{Conclusion}

Contacting thin foils of titanium grade 2 to a $\mathrm{CO}$ atmosphere at $800{ }^{\circ} \mathrm{C}$ for different times resulted in the formation of big grains of expanded h.c.p. $\alpha$ and an outer layer of NaCl type $\delta$. The c/a ratio of the h.c.p. structure of $\alpha$ increases from 1.588, for interstitially free h.c.p. $\alpha$, to 1.607 after 100 hours of exposure, along with an increase in the total interstitial content. The carbon and oxygen contents in the treated foils after 100 hours of exposure are $4.2(+/-0.34) \mathrm{wt} \% \mathrm{C}$ and $5.5(+/-0.33)$ wt $\%$ O, respectively, which would correspond to a mixture of $\alpha$ and $\delta$ in the calculated ternary Ti-C-O phase diagram. This lattice expansion of $\alpha$ phase is caused by the dissolution of both $\mathrm{C}$ and $\mathrm{O}$, but the exact ratio is presently undetermined. The hardness of $\alpha$ increases with the c/a ratio (composition) from 2193 (+/- 56.4) MPa for the untreated Ti to 11748 (+/- 313.75$)$ MPa after 100 hours.

\section{Acknowledgements}

The work has been carried out with financial support from the Danish Council for Independent Research under grant DFF - 7017-00182

\section{References}

[1] V. M. Fedirko, I. M. Pohrelyuk, and O. I. Yas'Kiv, Mater. Sci, vol 42, no. 3, pp 299-308, 2006.

[2] I. Pohrelyuk, O. Yaskiv, and V. Fedirko, Jom, vol. 59, no. 6, pp. 32-37, 2007

[3] N. Gammeltoft-Hansen, S. S. Munch, M. S. Jellesen, M. A. J. Somers, and T. L. Christiansen, Mater. Perform. Charact., vol. 6, no. 3, p. 231-43, 2017.

[4] L.F.S. Dumitrescu, M. Hillert, B. Sundman, A Reassessment of Ti-C-N based on a Critical Review of Available Assessments of Ti-N and Ti-C, Z. Met. 90 (1999) 534-541.

[5] Das, Karabi \& Choudhury, Pritha \& Das, Siddhartha. (2002). Journal of Phase Equilibria. 23.

[6] T. Christiansen and M. A. J. Somers, Metall. Mater. Trans. A Phys. Metall. Mater. Sci., vol. 37, no. 3, pp. 675-682, 2006. 
[7] T. S. Hummelshøj, T. L. Christiansen, and M. A. J. Somers, Scr. Mater., vol. 63, no. 7, pp. 761-763, 2010.

[8] T. L. Christiansen, K. Ståhl, B. K. Brink, and M. A. J. Somers, Steel Res. Int., vol. 87, no. 11, pp. 1395-1405, 2016.

[9] B. K. Brink, K. Ståhl, T. L. Christiansen, and M. A. J. Somers, J. Alloys Compd., vol. 690, pp. 431-437, 2017.

[10] ThermoCalc: [Online]. Available: https://www.thermocalc.com/media/50229/tcti1_extended_info.pdf.

[11] R. J. Hill and C. J. Howard, AAEC (now ANSTO) Report M112, Lucas Heights Research Laboratory, NSW, Australia, 1986

[12] Ogden, H.R. \& Jaffee, R.I., report, October 19, 1955; United States. 